Escuela de Ciencias Sociales y Humanidades, UNED, C.R. URL: http://investiga.uned.ac.cr/revistas/index.php/espiga/index ISSN: $1409-4002 \cdot \mathrm{e}-I S S N$ : 2215-454X

\title{
Ciencia, sexo y género
}

\section{María Elena León-Rodríguez}

Licenciada en Filosofía (Universidad de Costa Rica) y egresada de la Maestría en Filosofía (Universidad de Costa Rica). Actualmente, Profesora de Filosofía de la Escuela de Ciencias Sociales del Instituto Tecnológico de Costa Rica. Asociada al Colegio de Licenciados y Profesores en Letras, Filosofía, Ciencias y Artes. Instituto Tecnológico. Costa Rica. elena.leon.rodriguez@gmail.com

Recibido: 10 de febrero, 2016 - Aceptado: 12 de abril, 2016 - Corregido: 12 de agosto, 2016

\section{RESUMEN}

(Find the abstracts in English and French at the end of the article)

En este artículo se reseñan algunas posiciones teóricas que cuestionan el discurso científico que ha dado pie a la discriminación y al control de la corporalidad a partir de una noción de dualidad "natural" de los sexos. Se refiere, asimismo, a la intersexualidad como respuesta a la noción dualista de los sexos, y al sexismo y a la heteronormatividad como rasgos latentes del discurso científico.

Palabras clave: Feminismo, sexo, género, epistemología feminista, discurso científico.

"En todos los seres humanos tiene lugar una vacilación entre un sexo y el otro, y con frecuencia sólo las ropas mantienen la apariencia masculina o femenina, mientras, por debajo, el sexo es exactamente el contrario de que se ve en la superficie".

Virginia Woolf (1994, p. 139)

Recientemente, en Costa Rica, un error del Registro Civil dio pie al matrimonio de dos mujeres, aunque la legislación nacional prohíbe que dos personas del mismo sexo se casen (ver Murillo, 2015). Lo lamentable es que este acontecimiento fue producto de

Formato de citación según APA

León-Rodríguez, M.E. (2016). Ciencia, sexo y género. Revista Espiga. Vol. XV, (32), $137-144$

\section{Formato de citación según Chicago}

León-Rodríguez, María Elena. "Ciencia, sexo y género». Revista Espiga. XV. N. 32 (2016): 137-144 
un error registral y no una reivindicación de derechos humanos. Esa situación motivó lo que interesa discutir en este escrito: la pregunta acerca de lo que nos hace ser hombres o mujeres. ¿Será que el Registro Civil tiene potestad sobre la identidad sexual de los individuos o, más bien, que son caracteres de tipo biológico-neurológico u hormonal- los que influyen esa identidad sexual de las personas?

La imagen de los cuerpos se construye desde diferentes discursos de poder, a partir de los cuales se genera discriminación y control sobre la corporalidad, según el sexo. Esos entramados conceptuales han sido cuestionados por el feminismo, desde sus diversas vertientes, con el propósito de visibilizar ausencias y contradicciones que sostienen esos discursos de poder en tanto que prácticas de saber. La filosofía y la ciencia forman parte de esas prácticas que, en el peor de los casos, no han puesto en duda su entramado conceptual en materia de género. En virtud de lo anterior, es de radical importancia dirigir el cuestionamiento hacia esos ámbitos.

La indagación debe realizarse no solo a nivel personal o de la práctica diaria o en la cotidianidad, sino también a nivel de las instituciones que toman como fundamento teorías catalogadas como racionales y objetivas. Con todo, no hay que perder de vista el fundamento religioso que impera en los prejuicios y opiniones de las personas que elaboran el discurso científico. Además, en nuestros países, la religión es un elemento primordial y esto, en lugar de abrir espacios de discusión y análisis, empeora la situación de muchas personas con diversas orientaciones, dado que se asume el discurso religioso como una única fuente de verdad.

En consonancia con esta propuesta de cuestionar los discursos desde los cuales se construye la identidad sexual, el presente estudio reseña algunas posiciones teóricas que sustentan la crítica a la noción de dualidad natural de los cuerpos sexuados y al ejercicio de control sobre la corporalidad establecido por el discurso científico: la epistemología feminista, la indagación de la ciencia y la historia de las ideas científicas. Adicionalmente, se refiere a la intersexualidad como respuesta a la noción dualista de los sexos, y al sexismo y la heteronormatividad como rasgos latentes del discurso científico.

Tal como apunta la filósofa española Celia Amorós (1991), la razón debe ser crítica y criticarse a sí misma a través de una hermenéutica de la sospecha ${ }^{1}$, conceptualizada esta como una "actitud escéptica y crítica que ante el atajo facilón lo primero que se pregunta es ¿dónde está el truco?” (p. 74). Desde este punto de vista filosófico, es válido -y necesario- aventurarse a visualizar los trucos con los que se construyen los géneros y-por qué no- los sexos.

El punto de vista de partida son los estudios de ciencia, tecnología y sociedad (CTS)2, más exactamente, la epistemología feminista o fillosofía feminista de la ciencia, la cual "es

1. Para Alicia Puleo (2013), la hermenéutica de la sospecha "hace referencia a aquellas teorías que suponen que, por debajo de nuestra aparente racionalidad y voluntad libre, hay elementos que dirigen nuestra acción sin que lo advirtamos" (p. 2). Esta filósofa española se refiere a la hermenéutica de la sospecha en materia de género, y concluye sobre la necesidad de utilizar esta hermenéutica para implementar políticas de igualdad y desvelar el sesgo androcéntrico en la cultura.

2. Los estudios de ciencia, tecnología y sociedad (CTS) subrayan "el reconocimiento efectivo de la naturaleza social del sujeto cognoscente y de la empresa científica y, por otro lado, el análisis del conocimiento científico como una práctica en lugar de considerarlo un contenido acabado" (Adán, 2006, p. 39). 
la epistemología que se hace desde una perspectiva feminista o el feminismo que se usa como teoría crítica para enfrentarse a problemas epistemológicos o filosóficos" (Pérez y Ortega, 2014, 24). Esta corriente filosófica cuestiona la forma tradicional de hacer ciencia, ya que en muchas ocasiones la ciencia ha fundamentado estereotipos acerca de los géneros. Consiguientemente, desde esta posición, se examinan y critican las teorías que buscan diferencias cognitivas entre los sexos masculino y femenino, demostrando con ello la utilización de argumentos falaces, la falsa universalización de determinados hechos, la posición del sujeto cognoscente y la noción de objetividad aludida en la ciencia.

Dentro de esta misma corriente de pensamiento feminista -aunque con algunos puntos de vista y problemáticas distintos-se circunscribe la filósofa de la ciencia y feminista Helen Longino, quien planteó la necesidad de comprender el papel que desempeñan los valores en la construcción del conocimiento científico. Según Longino (1997), la ciencia tiene el hábito de fundamentar en su discurso los estereotipos de época acerca de los géneros; por ello propone una indagación de la ciencia, mediante la cual se revelen los mecanismos de construcción tanto del conocimiento como del género. Para realizar esta tarea, Longino se refiere al empirismo contextual, el cual "no trata la experiencia sensorial como la única legitimadora de las afirmaciones de conocimiento, dado que otros tipos de consideraciones podrían ser relevantes para establecer el contenido de la observación y la adecuada articulación de los datos” (p. 72). La justificación de las hipótesis científicas involucra la comparación de la hipótesis no solo con los datos observacionales, ya que existe una mutua relación entre el análisis crítico de los datos, las hipótesis y el trasfondo de las presuposiciones.

En esta línea, Longino analiza la investigación neuroendocrinológica de la conducta o, en otros términos, el estudio de la relación de las hormonas con la conducta. En relación con ello, examina la idea esgrimida por un grupo de científicos sobre el papel causal de las hormonas gonadales prenatales en el desarrollo de ciertas conductas propias del sexo o del género, a saber, las diferencias cognitivas entre hombres y mujeres, y la orientación sexual. Si bien es cierto que las hormonas gonadales determinan la capacidad reproductora de los seres humanos, mediante la diferenciación de los tejidos reproductivos que inicialmente son bipotenciales y luego se desarrollan en testículos u ovarios, no se infiere que determinen y programen el cerebro según el sexo. Realizar tal inferencia implicaría lo que Longino denomina un modelo hormonal lineal (p. 76), según el cual las explicaciones se plantean en términos de causalidades irreversibles y unidireccionales.

En contraposición a este criterio, existe la hipótesis de que el organismo humano responde a un ambiente no solamente endógeno sino también exógeno, y que el cerebro se auto organiza en vez de responder a instrucciones hormonales o genéticas; en esta línea, el ser humano se construye a sí mismo en un contexto psicosocial y político. Tras sus observaciones sobre este tipo de investigaciones, Longino enfatiza la necesidad de dar prioridad epistémica no solo a los datos observacionales presentados como evidencia, sino también al razonamiento que vincula a estos con las hipótesis causales en cuestión; y finalmente, llama la atención sobre la tendencia a generalizar a todas las especies -incluida la humana- los datos obtenidos de la investigación conductual en especies específicas de mamíferos. 
En esta misma línea que analiza el comportamiento y el factor biológico, la bióloga y filósofa estadounidense Anne Fausto-Sterling (2006) señala que la función génica puede comprenderse solo en el contexto de la célula misma o del microcosmos en el que se encuentra. Desde este punto de vista, el sistema nervioso y el cerebro tienen plasticidad; en otras palabras, los cambios anatómicos y fisiológicos de un ser humano no se limitan a la infancia y la adolescencia, sino que le acompañan a lo largo de toda su vida y evolucionan con la edad: "El cuerpo y las circunstancias en las que se reproduce no son entidades separables. De nuevo, algo que a menudo contemplamos como estático cambia a lo largo del ciclo vital, y solo puede comprenderse en términos de un sistema biocultural" (p. 290). Visto esto a la luz de lo reseñado hasta el momento, y recapitulando la propuesta de Longino, Fausto-Sterling afirma que usualmente los debates sobre biología del cuerpo son debates morales, éticos y políticos sobre la igualdad política y social.

Otra propuesta teórica para abordar el enfoque de género en el discurso científico es la del historiador y sexólogo estadounidense Thomas Laqueur (1990), en su libro La construcción del sexo: cuerpo y género desde los griegos hasta Freud, en el cual demuestra, basándose en pruebas históricas, cómo la ciencia se relaciona con las demandas de la cultura, cómo la explicación de la anatomía humana está vinculada a significados culturales y cómo el sexo solo puede explicarse dentro del contexto de las pugnas en torno al género y el poder. Según este autor, los progresos del siglo XIX en anatomía del desarrollo apuntan al origen común de ambos sexos en un embrión morfológicamente andrógino y sin diferencias intrínsecas. Durante muchos años, se creyó que las mujeres tenían los mismos genitales que los hombres, pero hacia 1800 los estudiosos se dieron a la tarea de fundamentar diferencias entre los hombres y las mujeres, apoyados -según ellos-en distinciones biológicas observables (p. 23).

¿A qué se debió ese emergente interés por jerarquizar y visualizar diferencias? Laqueur propone dos respuestas para esta interrogante: una de corte epistemológico y la otra de orden político. Según explica, las nuevas formas de interpretar el cuerpo se debieron en parte a que en el siglo XVII este ya no se consideraba un microcosmos, y la ciencia ya no generaba jerarquías de analogías sino que creaba corpus de conocimientos. Desde el punto de vista político, la disputa por el poder generó nuevas formas de constituir a los sujetos, y eso incidió sobre el orden social y la sexualidad. Tal como se puede colegir de ambas explicaciones, la corporalidad adquiere significados diferentes según los intereses políticos de la época, y esto muestra que "las nociones previas de diferencia e identidad determinan lo que se ve y lo que se cuenta del cuerpo" (p. 50).

Las propuestas teóricas reseñadas conducen al fin principal de este escrito: sustentar las bases para cuestionar la noción de dualidad "natural" de los cuerpos sexuados. Resulta indispensable debatir no solamente ese dualismo sino también las conceptualizaciones tradicionales de género e identidad sexual, porque condicionan las posibilidades de vida y reproducen las desigualdades de género. En relación con lo anterior, Fausto-Sterling (2006)declara que es la concepción de género y no la ciencia, la que puede definir el sexo de las personas, y que esa concepción de género afecta el conocimiento producido desde la ciencia en materia del sexo. Prueba de ello, según la autora, es el más reciente concepto de "intersexual", pues "para mantener la división de géneros, debemos controlar los cuerpos que se salen de la norma. Puesto que los intersexuales encarnan literalmente ambos sexos, su existencia debilita las convicciones sobre las diferencias sexuales" (p. 23). 
Cabe destacar que la práctica médica promueve el tratamiento de la intersexualidad con base en el criterio antes anotado, con el fin de mantener la clasificación binaria de los sexos. Según Fausto-Sterling, a la hora de asignar un sexo el personal médico se centra en la capacidad reproductiva o el tamaño del pene, lo cual demuestra que la elección de criterios tiene que ver más con lo social que con lo fisiológico. Asimismo, la autora indica que el tratamiento médico de las personas intersexuales debe cambiar, pues en algunos casos sería innecesario (por ejemplo, cuando no está en riesgo la vida del bebé) y porque la cirugía genital temprana conlleva muchas y muy importantes consecuencias, entre ellas, múltiples cicatrices, diversas operaciones y la eliminación de la capacidad orgásmica (p. 105). Como consecuencia de lo anterior, concluye que esa necesidad de la medicina de crear "los genitales apropiados" va en detrimento no solo físico, sino también psicológico de los pacientes y que, en lugar de ese procedimiento de asignación sexual, se debe proponer la posibilidad de que sea la propia persona, ya en edad adulta, quien decida qué hacer con su cuerpo y su identidad de género.

Desde el ámbito de la psicología, la psicóloga Silvia García (2014) enfatiza que el activismo intersexual desafía no solo la supuesta naturalidad del dualismo sexual, sino también la "natural" coherencia entre sexo, género y deseo heterosexual. Esta autora sostiene la existencia de no únicamente dos sexos preexistentes, sino una combinación múltiple de posibilidades de sexo cromosómico, hormonal, gonadal y morfológico ${ }^{3}$. En esta línea, la problemática acerca del sexo no es tan sencilla como en algunos casos se sugiere; más bien se cuestiona el dualismo sexual con miras a "desmontar nuestros filtros dicotómicos. El resultado es abrir espacios de posibilidad para que la variabilidad de cuerpos sexuados sea visible sin pagar el precio de la corrección de la diferencia” (p. 515).

Pensando en estas diferencias y recapitulando con Celia Amorós (2005), "quien está en posición de sujeto del discurso es el que mira y designa al otro. No se ve a sí mismo como diferente, sino como norma canónica" (p. 314). Desde este punto de vista, quien designa a un otro siempre lo hace desde su propia posición de poder, la cual, en nuestro entorno, usualmente corresponde aun sistema sexista y heteronormativo. Por este filtro pasa lo que se percibe como diferente y el sistema implica sojuzgar y violentar a quienes se salen de la norma. Esa percepción de la diferencia opera dentro de los discursos a través de una ideología que posibilita argumentos a favor de la discriminación de los "otros", sean estos mujeres, o bien intersexuales, homosexuales, lesbianas, entre otros grupos de sexualidades diversas. Sobre esa base, el sexismo y la heteronormatividad adquieren el monopolio del sentido e imponen un orden simbólico diferente para las personas según su sexo, género y orientación sexual.

En ese contexto sexista y heteronormativo, la diferencia se percibe como desigualdad; el dato biológico se interpreta desde un punto de vista que entiende la diferencia

3. De acuerdo con García (2014), la definición del sexo se compone "del sexo cromosómico, con su variabilidad cariotípica más allá del dualismo XX o XY; del sexo hormonal, con niveles diferentes tanto de las hormonas llamadas 'masculinas' como de las 'femeninas' en cada cuerpo, ya sea de varón o de mujer [sic]; del sexo gonadal, la diferenciación del tejido gonadal ovárico o testicular; del sexo morfológico, la morfología genital externa e interna (presencia del pene, clítoris, vagina, etc., con su variabilidad anatómica en cada cuerpo) y las características sexuales 'secundarias', vello, pechos, voz, etc.” (p. 503). 
como un disvalor. En contraste, la crítica al sistema heteronormativo conduce al cuestionamiento del dualismo sexual y a la apertura a la diversidad humana, y a rebatir la concepción de la diferencia como disvalor. Obviar la diversidad da pie a discriminaciones, y, si bien muchos países dicen legislar con base en los derechos humanos, todavía persiste la invisibilización de grupos humanos a quienes se les niegan esos derechos, discriminados principalmente por su orientación sexual y su identidad de género ${ }^{4}$. Hace falta más que una lista de decretos y leyes; se requiere un convencimiento propio de lo que nos hace seres humanos, antes que seres sexuados, descartando de paso la pretensión de un dualismo sexual.

En síntesis, es indispensable un cuestionamiento de aquellos discursos que se amparan en la objetividad y la racionalidad. Pues, tal como se explicó anteriormente, la ciencia, en tanto principal discurso racional-objetivo, suele utilizar argumentos falaces para justificar la universalización de ciertos hechos y de esta manera, sostener una dualidad "natural" de los sexos. De esta manera, desde la epistemología feminista de Longino(1997) se develó cómo la ciencia fundamenta su discurso desde estereotipos de género y, además, se explicó cómo el organismo humano responde a un ambiente no solamente endógeno sino también exógeno. Igualmente, a partir de las propuestas teóricas de Fausto-Sterling(2006) se demostró que los cambios anatómicos y fisiológicos corresponden a un sistema biocultural. Y, finalmente, desde la historia y la sexología, según el pensamiento de Laqueur(1990), se estableció la relación de la ciencia con las demandas de la cultura.

En este punto, cabe recordar la pregunta que se planteó al inicio de este escrito, ¿qué o quién tiene potestad sobre la identidad sexual de los individuos o, más bien, son caracteres de tipo biológico -neurológico u hormonal- los que influyen en esa identidad sexual de las personas? Pues a la luz de los argumentos presentados, el ser humano es más complejo que una célula, requiere ciertas relaciones sociales, culturales y políticas que lo hacen ser lo son. Por ende, la corporalidad adquiere significados diferentes de acuerdo con los intereses políticos de la época. En este punto, es importante resaltar la necesidad, ya señalada, de controlar los cuerpos que se salen de la norma y aquí es donde la intersexualidad cobra importancia, ya que en un intento desesperado de controlar cuerpos, la medicina promueve el tratamiento de la intersexualidad para mantener la clasificación binaria de los sexos. Ante esto, la psicóloga García (2014) abre la posibilidad de la existencia de no solamente dos sexos preexistentes, sino una combinación múltiple de sexos.

La pregunta de qué nos hace ser un hombre o una mujer debe quedar abierta y no se debe pretender basar la discriminación en lo biológico, pues tal como queda demostrado en este artículo, el ser humano es un ser complejo y la "humanidad" es algo que debe ser construido y pensado constantemente.

4. Según los Principios sobre la aplicación de la legislación internacional de derechos humanos en relación con la orientación sexual y la identidad de género (2007), más conocidos como Principios de Yogyakarta, "la orientación sexual se refiere a la capacidad de cada persona de sentir una profunda atracción emocional, afectiva y sexual por personas de un género diferente al suyo, o de su mismo género, o de más de un género, así como a la capacidad [de] mantener relaciones íntimas y sexuales con estas personas", y la identidad de género "se refiere a la vivencia interna e individual del género tal como cada persona la siente profundamente, la cual podría corresponder o no con el sexo asignado al momento del nacimiento, incluyendo la vivencia personal del cuerpo (que podría involucrar la modificación de la apariencia o la función corporal a través de medios médicos, quirúrgicos o de otra índole, siempre que la misma sea libremente escogida) y otras expresiones de género, incluyendo la vestimenta, el modo de hablar y los modales" (p. 6). 


\section{ABSTRACT}

\section{Science, sex and gender}

This article reviews some theoretical positions which question the scientific discourse that allows the discrimination and control of the body starting from the notion of "natural" duality of the sexes. It also refers to the intersexuality as an answer to the notion of sex duality, sexism, and heteronormativity as a suppressed characteristic of the scientific discourse.

Key words: feminism, sex, gender, feminine epistemology, scientific discourse

\section{RÉSUMÉ}

\section{Science, sexe et genre}

Cet article présente une critique de quelques positions théoriques qui mettent en question le discours scientifique qui a donné lieu à la discrimination et au contrôle de la corporalité à partir d'une notion de dualité naturelle des sexes. Il fait référence à l'intersexualité comme réponse à la notion duale des sexes, au sexisme et à l'hétéronormativité en tant que traits dormants du discours scientifiques.

Mots-clés: Féminisme, sexe, genre, épistémologie féministe, discours scientifique.

\section{Bibliografía}

Adán, C. (2006). Feminismo y conocimiento (trad. Iñaki Adán Villamarín). Galicia: SpiralMajor.

Amorós, C. (1991). Hacia una crítica de la razón patriarcal. Barcelona: Anthropos. (2005). La gran diferencia y sus pequeñas consecuencias... para las luchas de las mujeres. Madrid: Cátedra.

Fausto-Sterling, A. (2006). Cuerpos sexuados:la política del género y la construcción de la sexualidad (trad. Ambrosio García). Barcelona: Melusina.

García, S. (2014). La regulación tecnológica del dualismo sexual y el diseño de cuerpos normativos. En E. Pérez y E. Ortega(eds.) Cartografias del cuerpo:biopoliticas de la ciencia y la tecnología (pp. 469-519). Madrid: Cátedra.

González, M. (2001). Género y conocimiento. En J. López y J. Sánchez Ciencia, tecnología, sociedad y cultura en el cambio de siglo. (pp.347-358). Madrid: Biblioteca Nueva.

Gregori, N. (2014). "Llegar a ser mujer" Mujer/Hombre desde un diagnóstico de intersexualidad o ADS. En E. Pérez y E. Ortega, Cartografías del cuerpo. Biopolíticas de la ciencia y la tecnología. (pp. 469-519). Madrid: Cátedra.

Haraway, D. (1995). Ciencia, cyborgs y mujeres. La reinvención de la naturaleza. (trad. Manuel Talens). Madrid: Cátedra.

Harding, S. (1996). Ciencia y feminismo. (trad. Pablo Manzano). Madrid: Ediciones Morata. 
Keller, E. F. (2002). La paradoja de la subjetividad científica. En D. Fried Schnitman et. al. Nuevos paradigmas, cultura y subjetividad. (pp. 143-173). Buenos Aires: Paidós.

Laqueur, T. (1990). La construcción del sexo: cuerpo y género desde los griegos hasta Freud (trad. Eugenio Portela). Madrid: Cátedra.

Longino, H.E. (1997). Feminismo y filosofía de la ciencia. En M. González, J.A. López y J.L. Luján (eds.). Ciencia, tecnología y sociedad: lecturas seleccionadas (pp. 71-83). Barcelona: Ariel.

Miqueo, C. (2008). ¿Feminismo en el mundo científico médico?Isegoría. Revista de Filosofía Moral y Política, 38, 155-167.

Murillo, Á. (2015, 5 de noviembre). Un "error" permite el primer matrimonio gay en Centroamérica. El Pais(Sección Internacional). Recuperado de:http://internacional. elpais.com/internacional/2015/11/05/actualidad/1446679918_194388.html

Pérez, E. (1997). La retórica de la ciencia y el estudio de las diferencias sexuales. Theoría. Revista del Colegio de Filosofía, 5, 23-38.

Pérez, E. (2001). La perspectiva de género en ciencia y tecnología: innovación y nueva caracterización de las disciplinas. En J. López y J. Sánchez Ciencia, tecnología, sociedad y cultura en el cambio de siglo. (pp.283-296). Madrid: Biblioteca Nueva.

Pérez, E. (2008). Mitos, creencias, valores: cómo hacer más "científica" la ciencia; cómo hacer la "realidad" más real. Isegoría. Revista de Filosofía Moral y Politica, 38, $77-100$.

Pérez, E. y Ortega, E. (eds.). (2014). Cartografías del cuerpo:biopolíticas de la ciencia y la tecnología. Madrid: Cátedra.

Principios sobre la aplicación de la legislación internacional de derechos humanos en relación con la orientación sexual y la identidad de género (versión en español). (2007). Recuperado de:http://www.yogyakartaprinciples.org/principles_sp.pdf

Puleo, A. (2013). El concepto de género como hermenéutica de la sospecha: de la biología a la filosofía moral y política. Arbor Ciencia, Pensamiento y Cultura, 189(763), 1-10. doi: http://dx.doi.org/10.3989/arbor.2013.763n5007

Woolf, V.(1994). Orlando. Una biografía. (trad. Enrique Ortenbach). Barcelona: Lumen. 